\title{
DYNAMICS IN THE CENTRES OF TRIAXIAL ELLIPTICAL GALAXIES
}

\author{
Ortwin E. Gerhard \\ Max-Planck-Institut für Astrophysik \\ Karl-Schwarzschild-Str. 1 \\ 8046 Garching bei München \\ West Germany
}

\begin{abstract}
Orbits in the inner $k p c$ of a triaxial galaxy are discussed, taking into account the effect of a central density concentration like a massive black hole, a dense stellar nucleus, or a de Vaucouleurs-type cusp. Since the box orbits that form the backbone of a triaxial galaxy pass arbitrarily close to the centre after long enough time, they will eventually be subjected to large-angle deflections by a central point mass, and the triaxiality of the inner part of the system will thereby be destroyed. A $10^{8} M_{\odot}$ black hole is estimated to affect box orbits out to $1 k p c$ in a Hubble time, while a similar influence of the observed (extended) nucleus in M31 reaches out to $500 p c$ in the bulge. Regular box orbits persist, however, in systems with singular central density profiles such as implied by carrying the $r^{1 / 4}$ law all the way to the centre. This result can be approximately understood in terms of the frequency ratio $\Omega_{r} / \Omega_{\theta}$ remaining close to the harmonic value of 2 for many orbits in the corresponding spherical potential. Finally, I discuss observable consequences of the box orbit scattering process and future work, and use the presence of isophote twists in the central parts of a number of elliptical galaxies to obtain approximate upper limits on the masses of the black holes these systems may contain.
\end{abstract}

\section{INTRODUCTION}

While most existing galaxy models are assumed to have homogenous cores, giant elliptical galaxies often have central brightness profiles that appear to be still rising at the smallest presently resolvable radii (Schweizer 1979, Lauer 1985, Kormendy 1985). Moreover, they may contain massive black holes at their centres (LyndenBell 1969, Rees 1984), or dense stellar nuclei such as are observed in the bulge of M31 (Light et al. 1974) and probably in the bulges of many other nearby spiral galaxies (Kormendy 1985). It is therefore important to understand the dynamical effects of such features on the system as a whole.

In a spherical or axisymmetric potential, at least one of the components of the angular momentum vector is conserved, and so most stars are kept far away from the centre. Since the total mass of the central object is small compared to the mass of the core region of the galaxy (which is typically $\sim 5 \times 10^{9} M_{\odot}$ ), most stars in the cores of axisymmetric systems would also not be influenced much by the precise mass distribution at the very centre. However, there is increasing evidence 
for elliptical galaxies being in part triaxial systems (see Binney 1985 for a review, and Davies, this conference). In a triaxial galaxy the influence of a central compact object on the dynamics is much more profound, because a triaxial mass model depends critically on the existence and selective population of the box orbits, in particular the 'thin' box orbits (Schwarzschild 1979). As shown e.g. in Fig.1 of Gerhard \& Binney (1985; hereafter GB) these orbits go arbitrarily close to the centre after sufficient time, and different box orbits of the same energy differ at the centre by the direction of their velocity vectors. Any feature in the centre of the potential that can scatter the box orbits will therefore redistribute them in angular action (or in the size of their waists) and thereby affect the very heart of the system.

In the following I shall discuss three kinds of deviations from a homogeneous core: (i) a central point mass with a typical mass of $10^{8} M_{\odot}$, ie. $\sim 2 \%$ of the galaxy's core mass, (ii) a dense but extended central star cluster such as that inferred for M31, and (iii) central cusps as eg. implied by carrying a de Vaucouleurs profile all the way in to the centre (then $\rho \propto r^{-3 / 4}$ asymptotically, Young 1976). All of these features give rise to a complicated phase-space containing resonant or stochastic orbits in place of some or most box orbits. However, the first two are localized, and the results of the numerical orbit integrations can to a fair degree be described by a simple 'box orbit scattering' model. This will be described next; then I shall briefly consider effects on the global dynamics of the galaxy and the orbit structure of cuspy potentials, and finally describe some observational consequences and future work.

\section{BOX ORBIT SCATTERING}

Numerical orbit integrations show (GB; Figs. 2-4) that when a softened point mass with about 2 percent of the galaxy's core mass is placed at the centre of a triaxial galaxy and is gradually hardened, the box orbit region in phase-space first breaks up into regions occupied by families of resonant orbits and then becomes more and more irregular. In the presence of a hard point mass, most box orbits at low and intermediate energies become highly stochastic. Inside the core radius some box orbits are transformed into loops; of the box orbits reaching out to several core radii only a small fraction can avoid disruption by being trapped in orbital resonances. Stochastic orbits at these energies can be described as sequences of segments of regular box orbits, separated by discrete scattering events.

These numerical results may be approximately understood by the following simple model. Denote the core mass of the underlying galaxy potential by $M_{c}$ and the mass of the central compact object (CCO) by $m$; then for a typical $\mu \equiv$ $m / M_{c} \simeq 0.02$ the radius of influence of the CCO - at which the forces due to the galaxy and the CCO are equal - is $r_{h} \simeq \mu^{1 / 3} r_{c} \simeq 0.3 r_{c}$, with $r_{c}$ being the galaxy's core radius. Now imagine that stars from the galaxy enter the radius of influence $r_{h}$ with a velocity $v_{h}$ set by the underlying galaxy potential, and that inside $r_{h}$ they may be considered to be on hyperbolic Keplerian orbits with asymptotic velocity $v_{h}$. Then large-angle scattering occurs for impact parameters

$$
b<b_{l}=\frac{G m}{v_{h}^{2}}=\mu r_{c}\left(\frac{v_{c}}{v_{h}}\right)^{2},
$$

where $v_{c}$ is the circular velocity at the edge of the core. Empirically, one finds that box orbit scattering by a point mass becomes effective in destroying the regularity 
of an orbit when

$$
b \leq 3 b_{l},
$$

and by an extended nuclear star cluster when in addition the half-mass radius is

$$
r_{1 / 2} \leq 2 b_{l}
$$

The latter condition corresponds approximately to the condition $v_{n}^{2} / v_{h}^{2} \leq 5$, where $v_{n}$ is the characteristic velocity of the nucleus. Since box orbits come arbitrarily close to the centre after sufficient time, equation (2) implies that even a very small point mass in the centre will ultimately destroy the triaxiality of the entire system.

Thus the problem becomes one of time-scales. To estimate how long it takes a star on a given box orbit of specified energy to come sufficiently close to the centre, one needs to know the probability for it to go anywhere through the orbit's waist (i.e. the section $x=0$ through the orbit). The simplest assumption is that the probability of going through any point in the orbit's waist is independent of where this point lies in this section; then $N(<b) \propto b^{2}$. The numerical integrations in GB verify the analogous assumption for planar orbits, $N_{p l}(<b) \propto b$, and in this case also the amplitude of $N_{p l}(<b)$ as determined by a suitable average over the angular actions for the box orbit segments agrees very well with the numerical results. Then one expects a star to be scattered after about (area of the waist) $/ 9 \pi b_{l}^{2}$ passages through the centre, or (in the simplest case) after time

$$
\tau_{l}=\frac{\left(2 r_{c}\right)^{2}}{9 \pi b_{l}^{2}} \tau_{d y n} \propto \mu^{-2}\left(\frac{v_{h}}{v_{c}}\right)^{4} \tau_{d y n}
$$

$\tau_{l}$ is thus proportional to $\mu^{-2}$, to the dynamical time, and to a dimensionless velocity factor that describes the energy of the orbit in a given mass model of the galaxy. Both this factor and $\tau_{d y n}$ increase with energy; thus equation (4) shows that for any value of the mass ratio $\mu$, there is a maximum radius out to which box orbit scattering is effective, and, conversely, for any radius there is a minimum mass required for scattering in a Hubble time.

Assuming that the inner parts of the galaxy are approximately described by a modified Hubble law, one finds in this way that in a typical triaxial elliptical galaxy a central black hole of $\sim 10^{8} M_{\odot}$ will scatter box orbits that reach out to $\sim 1 k p c$ (GB). In the case of the extended nucleus of M31 (according to the Stratoscope II observations of Light et al. 1974 and the rotation measurements of Walker 1974, but see Kormendy, this conference) the maximum radius for box orbit scattering is not determined by the time-scale, but through the softness condition (3) (very approximately), and is numerically found to be $\sim 500 p c$ (Gerhard 1986).

These results remain unchanged if the figure of the galaxy rotates slowly about its smallest principal axis. In a tumbling potential the closed long-axial orbits that sire the box orbit family become prograde orbits which no longer pass exactly through the centre of the system. The distance at which these orbits pass the centre as they cross the intermediate axis of the potential, increases both with orbital energy and with the angular speed at which the potential rotates. However, rotation can only prevent the dissolution of the triaxiality of the system by the box orbit scattering process if a substantial part of the box orbit family never comes close to the centre. I.e. the offset of the closed long-axial orbit along the intermediate axis has to be at least of order the maximum width of a box orbit 
in the absence of figure rotation or black hole. From this condition one estimates (GB) that only box orbits reaching out to at least a quarter of the corotation radius can escape being scattered, so that figure rotation cannot prevent the dissolution of box orbits in the inner few core radii of a slowly rotating elliptical galaxy, or in the inner 500pc of M31's bulge (which has an estimated corotation radius of at least $5 k p c$, cf. Gerhard 1986).

\section{EFFECT OF BOX ORBIT SCATTERING ON THE GALAXY}

The scattering of the box orbits by a compact object in the centre of a triaxial galaxy will tend to establish a uniform distribution of box orbits in their relevant angular action. Then the selective population of the thin box orbits cannot longer be maintained and hence the CCO must ultimately destroy the triaxiality of the system. What is less certain is whether the disruption of triaxiality occurs abruptly, or whether it proceeds gradually from the inside out. The latter possibility arises because the disruption time-scale (4) is a rapidly increasing function of energy, and so in a fixed potential the low-energy box orbits are scattered much before the highenergy ones and axisymmetry may spread gradually from the centre. However, if collective effects due to the dissolution of the box orbits in the core of the system are strong, they may alternatively lead to a rearrangement of a large part of the system relatively quickly.

To settle this question will require reliable $\mathrm{N}$-body models of triaxial galaxies with a genuine central point mass. A first attack on this very difficult numerical problem was made by Norman, May \& van Albada (1985); their simulations indicate, however, that individual orbits can currently not be integrated with sufficient accuracy for one to have confidence in results obtained from $\mathrm{N}$-body simulations for this problem (see also van Albada, this conference).

The following simple argument suggests, however, that triaxiality will be lost gradually, from the inside out, rather than in the manner of an abrupt phase change. If one compares a prolate stellar system of constant ellipticity with one in which only the outer parts are prolate, but which has a spherical core, then the quadrupole moment in the outer parts is not very different between the two systems. Since it is this quadrupole moment (i.e. the tangential force) which every few dynamical times reverses the angular momentum of stars on box orbits, this suggests that at any time the galaxy will approach axisymmetry only at those radii at which the disruption time (4) is less than its age, and remain triaxial at larger radii.

\section{CENTRAL DENSITY CUSPS}

In recent years there has been much discussion of whether the stellar densities in the centres of elliptical galaxies continue to rise at the smallest radii that can be resolved from the ground, or whether the density finally flattens off in the very inner parts of the system (e.g. Schweizer 1979). In view of the ability of CCO's to destroy the triaxiality of at least the inner parts of the galaxy, it is therefore interesting to know how pronounced a density cusp has to be before it can have a similar effect. GB investigated the orbital structure in the equatorial planes of triaxial potentials, that correspond to power-law density distributions in the ellipsoidal radius a (actually, they studied orbits in the planes of prolate systems containing the respective long axis, but the difference to the triaxial case is small). A particular such case is that 


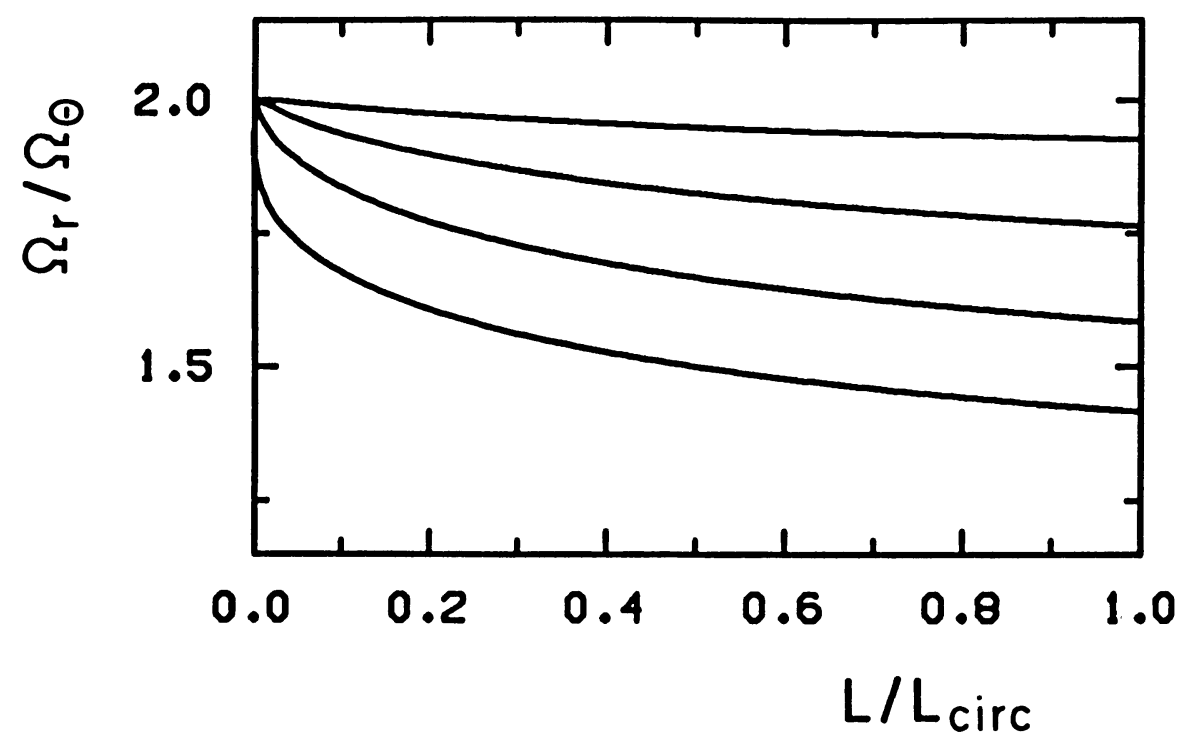

Fig.1: The frequency ratio $\Omega_{r} / \Omega_{\theta}$ in a number of spherical potentials corresponding to density profiles $\rho(r) \propto r^{-\gamma}$, as a function of the angular momentum of the orbit normalized to that of the circular orbit of the same energy. Curves are (from top) for $\gamma=0.3, \gamma=0.9, \gamma=1.5, \gamma=2.0$ (scale-free logarithmic potential).

obtained by carrying the de Vaucouleurs profile all the way into the centre; then for small $a$

$$
\rho(a)=\rho_{0} a^{-p}, \quad a^{2}=x^{2}+\frac{y^{2}+z^{2}}{q^{2}}
$$

and $p=0.75$ (Young 1976). In this case, both the force and the circular velocity tend to zero at small a, and the corresponding potential contains box orbits at all energies of interest, besides containing also a few resonant orbit families and some stochastic orbits. Indeed, the fraction of phase-space occupied by the box orbits is substantially larger in this potential than in Schwarzschild's (1979) potential at both low and high energies. Thus there is every reason to believe that self-consistent triaxial galaxy models can be constructed which have a de Vaucouleurs brightness profile.

The existence of box orbits in potentials with singular density profiles can be related to the range of values of the frequency ratio $\Omega_{r} / \Omega_{\theta}$ in the corresponding spherical potential. Fig. 1 shows this ratio for a number of spherical potentials, as a function of the orbit's angular momentum normalized by the angular momentum of the circular orbit. In a harmonic potential, $\Omega_{r} / \Omega_{\theta}=2$ for all orbits; in the case corresponding to $\rho \propto r^{-\gamma}$ it is near the harmonic value for almost all orbits when $\gamma=0.3$, and for many orbits still when $\gamma=0.9$. Only when the potential approaches the scale-free logarithmic potential $(\gamma=2.0)$ is $\Omega_{r} / \Omega_{\theta}$ significantly different from 2 already for orbits with quite low angular momenta. Correspondingly, most of the phase-space in a genuinely scale-free logarithmic potential is occupied by loop 
orbits and by (in $x-y$ ) 1:2, 3:2, and 4:3 resonant orbits families, while box orbits play little or no role; cf. GB.

\section{OBSERVABLE CONSEQUENCES, BLACK HOLE MASSES, FUTURE WORK}

We have argued above that if box orbit scattering due to a central massive black hole or dense star cluster occurs in a triaxial galaxy, it will gradually axisymmetrize the system from the inside out. Then if the galaxy started out with constant ellipticities, it should now appear rounder in its inner region as compared to its outer parts. However, there is nothing that prevents a galaxy from being made with a rounder core in the beginning, so that interpreting such observations is inherently ambiguous. Nevertheless it is interesting to notice that galaxies which are fairly round inside a few core radii and become more flattened further out are common, eg. in Lauer's (1983) sample. Among his 42 galaxies, there are 21 which are consistent with having no isophote twist in the region outside of 4". Of these 10 have ellipticity profiles that rise from being nearly round at 4 " to $\epsilon \sim 0.3$ further out (both before and after seeing corrections). This pattern is consistent with these systems being triaxial galaxies seen from near one of their principal planes, or with them being axisymmetric objects without constraint on the projection geometry. A Monte Carlo-type statistical analysis is needed to see whether one could rule out on probability grounds the hypothesis that the inner parts of these galaxies are triaxial. If so, this would be interesting from the point of view of the scattering process.

The argument may be turned around in the following way. If a galaxy shows isophote twists at some small radius $a$ which are not caused by dust, and if one assumes that these are due to triaxiality and imply the existence of box orbits of scale $a$, one may set an approximate upper limit to the mass of the black hole this galaxy could contain, by estimating the box orbit disruption time and requiring that it should exceed a Hubble time at scale $a$. This has been done for the galaxies in Lauer's sample that show clear isophote twists outside 4" and for which the seeing correction left the position angle profiles nearly unchanged. Since the photometry of these galaxies is in the red, the effects of dust should not play a too important role, but some galaxies were discarded on Tod Lauer's advice. In Table 1 I list for the remaining galaxies with near-central twists (names in column 1) the radius at which the twist occurs (col. 2), the magnitude of the twist (col. 3), the ellipticity in the region of the twist (col. 4), the core radius (cols. 5,6), the line-of-sight velocity dispersion (col. 7), and the upper bound inferred for the mass of the central compact object (col. 8). This was obtained by equating to a Hubble time a more accurate expression for the box orbit scattering time $\tau_{l}$ (equation 25a of GB), assuming that the twists are due to box orbits at the scale of the twists. Typical upper limits range from a few $10^{7}$ to a few $10^{8}$ solar masses. If, less conservatively, one included a galaxy like NGC 1052 in the list, which may have a small twist at $3 "$, one would derive $\sim 9 \times 10^{6} M_{\odot}$ on the same assumptions. With the very best multicolour photometry one might push the limit down to $\sim 2 \times 10^{6} M_{\odot}$. if isophote twists due to triaxiality were seen at one core radius of $\sim 1$ " $\sim 100 p c$.

On the theoretical side the most uncertain part is the collective response of the system as a whole to the box orbit scattering and subsequent evolution of its inner parts. The study of this important problem will require sophisticated $\mathrm{N}$-body simulations, possibly making use of a hybrid approach that treats orbits in the vicinity of the central object specially. Alternatively, one might use that 


$\begin{array}{cccccccc}\text { NGC } & \text { at/" } & \Delta \phi /^{0} & \epsilon & \mathrm{rc} / " & \mathrm{rc} / \mathrm{pc} & \sigma / k m s^{-1} & M / M_{\odot} \\ & & & & & & & \\ 596 & 6-9 & 20 & .05 & 0.9 & 83 & 180 & 4 \times 10^{7} \\ 636 & 6-10 & 12 & .06 & 1.39 & 128 & 186 & 5 \times 10^{7} \\ 741 & 4-5 & 10 & .15 & 1.91 & 519 & 309 & 2 \times 10^{8} \\ 1407 & 4-6 & 15 & .05 & 3.21 & 247 & 288 & 4 \times 10^{7} \\ 1453 & 5 & 5 & .12 & 1.38 & 285 & 280 & 2 \times 10^{8} \\ 5846 & 4-16 & 20 & .04 & 2.51 & 292 & 255 & 7 \times 10^{7} \\ 7626 & 4-18 & 9 & .10 & 1.1 & 206 & 275 & 2 \times 10^{8} \\ 7768 & 7-10 & 5 & .20 & 1.11 & 467 & 260 & 9 \times 10^{8} \\ & & & & & & & \\ 1052 & 3 & 10 & .20 & 1.08 & 75 & 207 & 9 \times 10^{6} \\ 4474 & 2-4 & 5 & .07 & 3.70 & 282 & 317 & 3 \times 10^{7} \\ 4649 & 3 & 10 & .05 & 3.41 & 260 & 361 & 2 \times 10^{7} \\ 7785 & 3 & 8 & .25 & 1.12 & 226 & 241 & 5 \times 10^{7}\end{array}$

Table 1: Upper limits to the mass of a possible central black hole in a number of ellipticals, obtained from relating the scale of observed isophote twists to the corresponding box orbit scattering time-scale. See text for details. The first part of the table includes only galaxies with twists outside $4^{\prime \prime}$, while the second part lists a few galaxies with apparent twists at $\sim 3^{n}$.

the evolution at each radius occurs on a time-scale much longer than the dynamical time-scale, so that the action variables are conserved except in the scattering events. Then, even though each star changes its angular action substantially when being scattered, the distribution function of the angular action may still be considered as slowly varying in a known way, so that the change in the mass distribution could be computed iteratively.

\section{REFERENCES}

Binney, J.J., 1985. Mon. Not. R. astr. Soc. $212,767$.

Gerhard, O.E., 1986. Mon. Not. R. astr. Soc. $219,373$.

Gerhard, O.E., Binney, J.J., 1985. Mon. Not. R. astr. Soc. 216, 467.

Kormendy, J., 1985. Astrophys. J. (Letters) 292, L9.

Lauer, T.R., 1983. PhD thesis, University of California, Santa Cruz.

Lauer, T.R., 1985. Astrophys. J. 292, 104.

Light, E.S., Danielson, R.E., Schwarzschild, M., 1974. Astrophys. J. 194, 257.

Lynden-Bell, D., 1969. Nature 223, 690.

Norman, C.A., May, A., van Albada, T.S., 1985. Astrophys. J. 296, 20.

Rees, M.J., 1984. Ann. Rev. Astr. Astrophys. 22, 471.

Schwarzschild, M., 1979. Astrophys. J. 232, 236.

Schweizer, F., 1979. Astrophys. J. 233, 23.

Walker, M.F., 1974. Publs. astr. Soc. Pacif. 86, 861.

Young, P.J., 1976. Astron. J. 81, 807. 


\section{DISCUSSION}

Norman: Two points. Firstly, the stochasticity produced by the black hole may help provide fuel for the hole. Unpublished calculations done with Andrew May indicate that stochasticity is significant in the nucleus with $0.001<M_{h} / M_{\text {core }}<0.1$ with the upper limit occurring because the hole dominates the nucleus and produces regular orbits again. This could be very important for luminosity evolution. Secondly, although as probably will be discussed by Tjeerd van Albada, the simulation we made was very difficult and needs to be greatly improved, the result was a significant roundening of isophotes by one or two ellipticity subclasses in a Hubble time. Radio galaxies are seen to be rounder-the latest on this is in a poster at this meeting. Although this occurs at larger radii than seen in our simulation, the effects may well be correlated.

Gerhard: To your first point: The cross section for tidal disruption of stars by the hole is always much smaller than the cross section for box orbit scattering, so that the triaxiality of the core, and the stochastic orbits introduced by the hole in it, disappear quickly. Consequently, the feeding rate is a rapidly decreasing function of time, and it seems unlikely to me that triaxiality can help feed powerful radio sources-let alone quasars - for longer than $\sim 10^{6} \mathrm{yr}$, even with very massive black holes (cf. Figure 9 in Gerhard \& Binney, 1985, Mon. Not. R. astr. Soc., 216, 467). To the second point: I understand that there is some controversy about the observational effect you mention, but if radio galaxies are rounder at $R_{25}$ than normal galaxies, very massive black holes and very small core radii are required if this is to be explained in terms of the box orbit scattering process. But I agree that it is important to understand better the collective effects that the changes in the inner $\mathrm{kpc}$ may induce.

Lake: Begelman, Blandford and Rees (1984, Rev. Mod. Phys., 56, 255) argue that radio galaxies emit at small fractions of the Eddington limit. NGC 1052 is a radio galaxy and is the galaxy whose black hole mass is most strongly constrained by your method. How does the mass from the Eddington limit compare?

Gerhard: NGC 1052 belongs to a class of compact-active E/SO galaxies for which $10^{40} \mathrm{erg} / \mathrm{s}$ is a characteristic luminosity (Jones, Wrobel \& Shaffer, 1984, Astrophys. $J ., 276,480$ ). If this is $1 \%$ of the Eddington limit, the inferred mass would be $\sim 10^{4} M_{\odot}$. 